\title{
Assessing customs performance in the Mediterranean ports. KPI selection and Best practices identification as part of the MEDNET project
}

\author{
Pau Morales Fusco \\ Senior Researcher, CENIT - Centre d'Innovació del Transport \\ Sergi Saurí Marchán \\ Director, CENIT - Centre d'Innovació del Transporty \\ Anna-Maria Lekka \\ National Technical University of Athens, Schoool of Civil Engineering, Department of \\ Transportation Planning and Engineering \\ Iosif Karousos \\ National Technical University of Athens, Schoool of Civil Engineering, Department of \\ Transportation Planning and Engineering
}

\section{SUMMARY}

The seamless flow of goods, people and investments across the Mediterranean necessitates a well-functioning port and transport system. More efficient port operations enhance seamless logistics and promote safety, efficiency, interoperability and interconnectivity of transport networks in the Mediterranean area.

To promote the exchange of knowledge and expertise relevant to port and customs procedures and simplification of clearance for vessels and cargoes in the Mediterranean, the MEDNET project was launched. As part of the project a common evaluation framework for the performance of ports in the form of a set of Key Performance Indicators (KPIs) together with a list of the best practices in terms of operations and customs procedures was developed.

In total, 50 ports were analyzed and given a KPI regarding traffic, financial, operational and human resources, sustainability and customs procedures. The values were latter crossed with the current good practices on operation in terms of customs procedures. And a small correlation between KPIs performance and implantation degree of good practices in customs procedures was found. This presentation exposes the methodology to assess the ports' performance and the best practices identification.

\section{INTRODUCTION}

The seamless flow of goods, people and investments across the Mediterranean necessitates a well-functioning port and transport system. More efficient port operations enhance seamless logistics and promote safety, efficiency, interoperability and interconnectivity of transport networks in the Mediterranean area.

In that context, the MEDNET project was launched to establish and operate a network of port authorities and transport experts in the Mediterranean region, focusing on the exchange of 
knowledge and expertise relevant to port and customs procedures and simplification of clearance for vessels and cargoes. This is expected to improve the common understanding and promote the introduction of information systems to ports operation and potentially to other intermodal modes.

Multiple measures to streamline freight going through port terminals and, therefore, increase the competitiveness of the maritime system, have been adopted through the years. Some, to enforce regional, national or European regulations (like the recent adoption of the Directive 2010/65 on the implementation of Single Window in Customs), others spawning from the initiative of port authorities. In any case, the effectiveness of such initiatives, would be hard to quantify, unless they can be checked against a set of performance indicators closely related to what has been implemented.

With this idea in mind, part of the project involved the identification of good practices in the Mediterranean Ports to ease the procedures and assess their effects on the port performance in order to identify the families of practices necessary in any 'ideal port'. This paper proposes a set of key performance indicators (KPIs) to later construct a framework looking for a link between practices and performance.

With this idea in mind, a comprehensive list of current practices that could be labeled as good or best practices in 60 ports from 9 countries from the Mediterranean were identified. The goal of this paper being to divulgate the findings made, digest the practices and look for an hypothetical connection between their implantation and the current performance of ports with the aim to identify the best practices that are necessary in any 'ideal port'. To do so the paper proposes a set of key performance indicators (KPIs) and links the values obtained with the level of 'good-practicing' of each port by using a DEA (Data Envelopment Analysis) model.

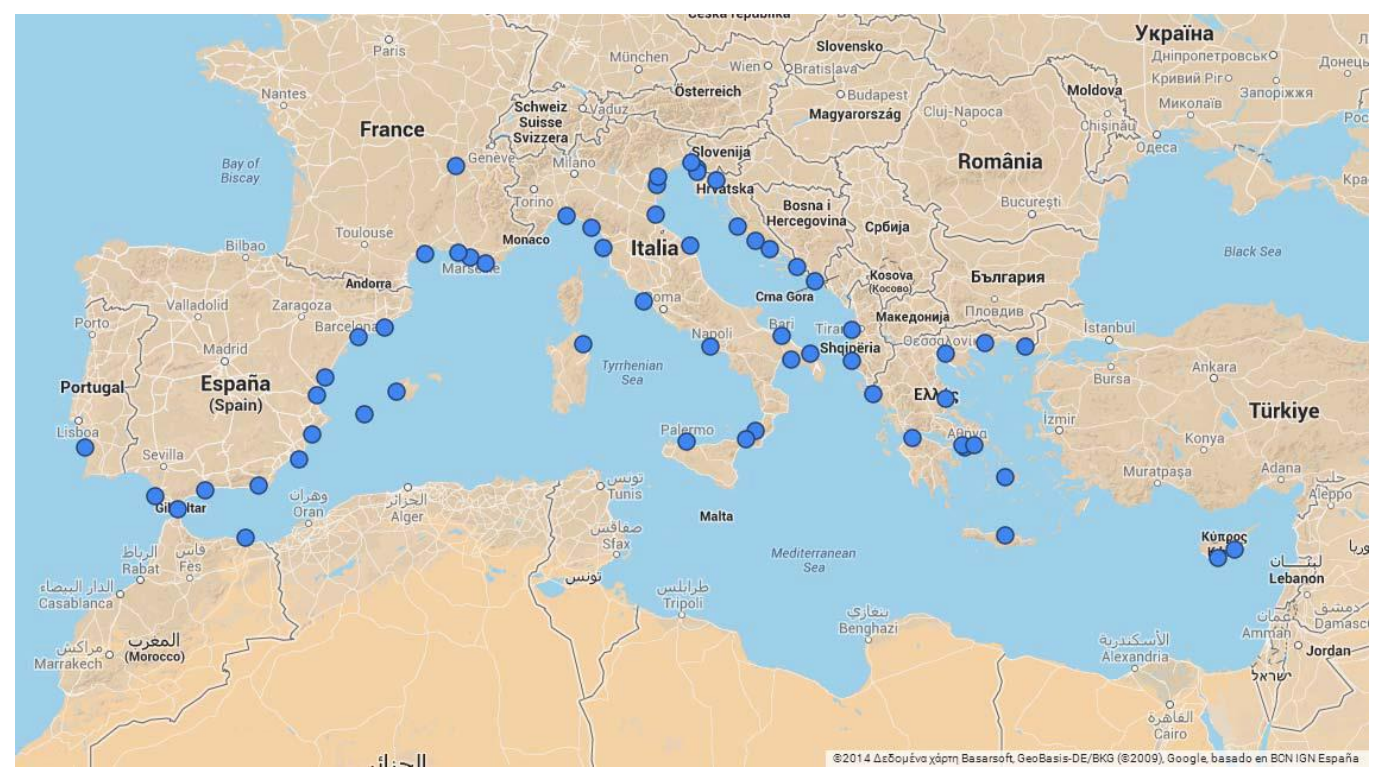

Fig 1. Map with ports analysed

The first block of this paper discusses the major precedents on port performance assessment methodologies to, in a second block, introduce a set of KPIs to grade them, explaining how the selection was made and the results obtained after calculating them for a selection of ports. A 
third block identifies the families of good practices currently in operation at ports to, in the fourth section, propose a methodology to link KPIs and good practices observations. The results from applying it to the sample of studied ports and conclusions and further developments to be pursued are discussed at the end.

\section{PERFORMANCE BENCHMARKING AT PORTS. A LITERATURE REVIEW}

Benchmarking is understood as the systematic comparison of the performance of one port against other ports. The idea is to compare entities with the same framework of properties, or in this case, procedures, with the outputs -performance- achieved from their implementation. The production entities could be firms, organizations, divisions, industries, projects, decision making units, individuals or, in this case, ports.

A traditional way to overcome some of the difficulties of making rational ideal evaluations is to use what practitioners call Key Performance Indicators, KPIs. These numbers are supposed to reflect in some essential way the purpose of the firm. Generally speaking, KPIs are an essential tool for organizations to understand and monitor the quality of their performance in relation to their strategic goals and objectives. More specifically, KPIs try to reduce the complex nature of organizational performance to a small number of key indicators in order to control, monitor and improve the quality of their services.

The foundation of benchmarking performance and KPI values for ports was set up in 1976, when the United Nations Conference on Trade and Development (UNCTAD, 1976) published a list of port performance indicators, which has been established as a well-recognized reference point for guiding researchers ever since. The suggested port performance indicators were divided in two broad categories:

- Financial, which quantifies aggregate port impacts on economic activity

- Operational, which evaluates input/output ratio measurements of port operations, in relation to productivity and effectiveness measures.

Later approaches, however, decided to complement this first set of KPIs with indicators that also considered qualitative aspects. Talley (1994), for instance, proposed a set of indicators to evaluate a port's performance considering the economic optimum beyond engineering parameters and considering the port's price and operating options (for differentiating service).

More recently, Key Performance Indicators have been obtained according to the current status and needs of ports, such as including concepts like the logistics services being offered, adding qualitative aspects to be measured. As an indication, Owino et al. (2006) produced an updated list with 30 different performance indicators. Marlow and Paixão (2003), after highlighting the importance of measuring port effectiveness related with leanness and agility in port operations, suggested a set of new indicators to visualize, within the port environment, the entire logistics transport chain. Finally and in the same context, Bichou and Gray (2004) based performance on logistics services, and argued that all performance indicators can be reduced to three broad categories, physical, productivity; and economic and financial indicators. 
The use of port performance indicators in the port industry has increased in recent years. Various port authorities have developed and reported port performance indicators in their annual reports. However, clear standards and a common ground with the same data definition is missing. These are required for both the development of aggregated performance indicators (e.g. for a whole country) and the allowing benchmarking between ports.

Some ports with their own KPIs systems. For instance, the Port of Rotterdam uses 32 KPIs to grade port operations and assess the current quality of the services. Similarly, the Port of Hamburg, in the framework of project StratMoS, developed three sets of indicators -depending on the point of view of the stakeholder being involved- to assess port performance, qualitatively, and depending on the user considered. The system is usually automated and can be checked dynamically, for instance, the Port of Venice developed the LogIS system to follow up how several KPIs perform.

However, to make a good use of the Key Performance Indicator, KPI, it is necessary to use it comparatively. For instance, if our port just had one input to produce one output and we knew the input-output data from several ports, we could use this to determine who is doing best by simply comparing the numbers, in what is often called productivity, i.e. output per input.

The direct use of KPIs has two main drawbacks:

- When we compare firms (ports) with highly different outputs we are assuming constant returns to scale, which is not likely the case in our assessment.

- The evaluations are partial. One KPI may not fully reflect the purpose of the firm. We need an evaluation taking into account several output-input ratios simultaniously. By doing so it is not possible to straight-forward identify the most productive firm (port).

There are some methodologies addressing those drawbacks. As a reference, the books by Bogetoft \& Otto (2010) or Cooper, Seiford, \& Tone (2007) give an overview of benchmarking techniques, focused in the Data Envelopment Analysis method (DEA) and its application through the accompanying software.

Tongzon (1995) was the first to link practices and performances in a port environment. After empirically determinate the main factors affecting the performance of 23 international ports, two performance indicators were considered and a set of distinct factors (or determinants) per each indicator. The performance and effect of each determinant over the indicator was determined by means of a two-stage least squares estimation procedure. Despite the results were far from conclusive, what is interesting is that the study pointed out on the necessity to consider the individual efficiency of terminals when assessing port performance and the difficulty in quantifying delays. Issues both, encountered when doing this research.

At an European level, Cullinane \& Wang (2006) provide a relevant study, mainly for its scope (69 port container terminals from 24 European countries). The authors used a DEA analysis with constant returns to scale (CCS) and also variable returns to scale (VRS) and besides finding out that the majority of terminals were low performers, identified returns to scale in their 
performance and that the geographical area also influences the performance of ports.

In terms of DEA, the research done by Panayides, Maxoulis et al. (2009) was of special interest since provided a comparative and critical analysis of the use of DEA in port benchmarking from the nature of its application regarding orientation, use of returns to scale to the variables used and their definition. The research states that better results are obtained with panel data covering several observations for any given port than using a larger set of ports or variables, whose number should be kept as small and as representative as possible. Otherwise, too many ports may be qualified as peers. That is, qualified as efficient because there are not counterparts to be compared with them.

Finally, the work by Tovar \& Rodríguez-Déniz, (2015) not only provides a good overview of the benchmarking techniques for efficiency (productivity) assessment in ports but also highlights the necessity to produce clusters of ports with similar characteristics to avoid confusions between inefficiency and heterogeneity. Interestingly, the study assesses different methods used for benchmarking and port clustering, either non-parametric models (DEA) or parametric techniques (Stochastic Frontier Analysis, SFA).

\section{KPI CONSTRUCTION AND GOOD PRACTICES IDENTIFICATION}

\subsection{KPI Construction}

An initial set of KPIs was identified and classified into six main categories: traffic; financial; operational; customs procedures; sustainability and security; and human resources. The Delphi method was used for the selection of the final set of KPIs and reference values, based on the experience and expert opinion of the MEDNET consortium partners. The initial set of 77 different KPIs was successfully reduced to a more reasonable number of indicators, 27 (Table 1), also in line with the availability of relevant data in the Mediterranean ports. 


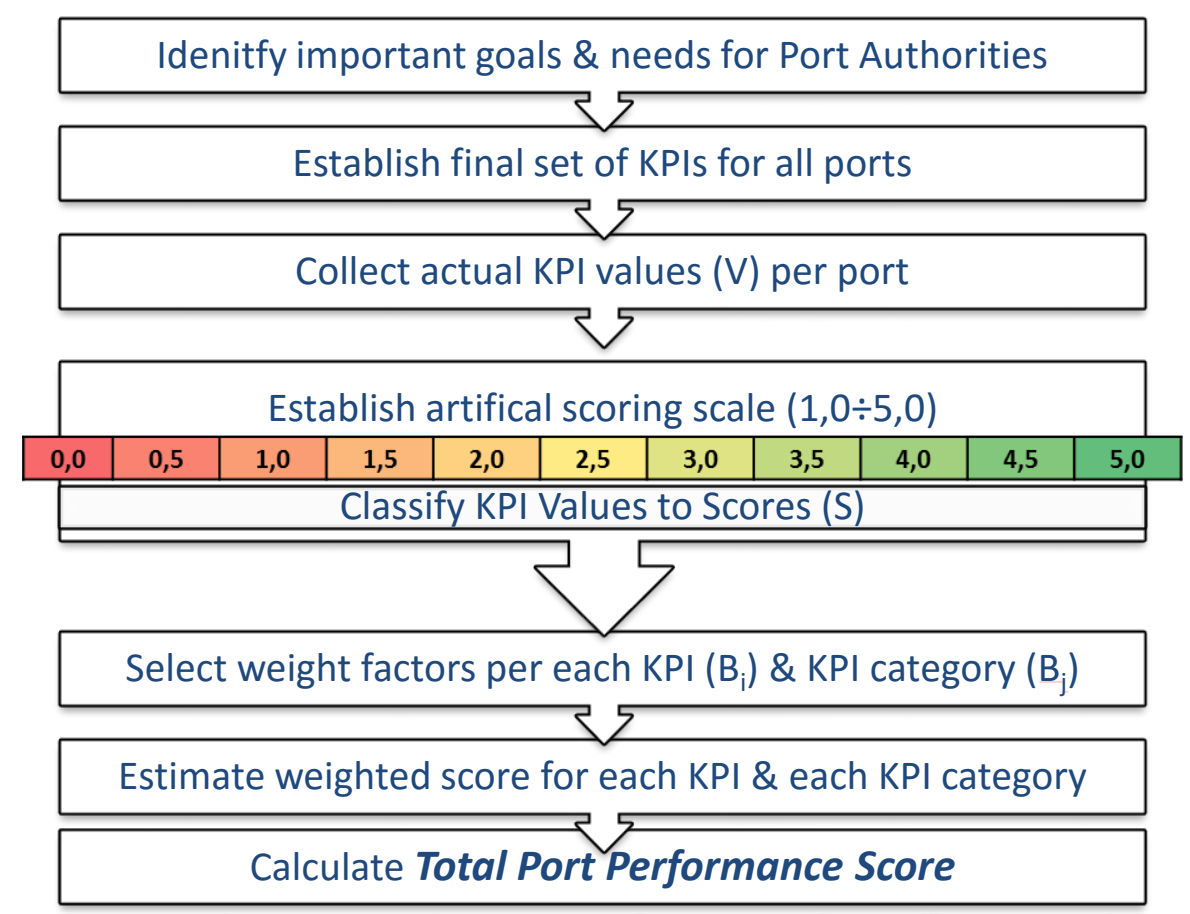

Fig 2. KPI definition. Methodology flowchart

The methodology for assessing the ports performance in terms of their everyday operations and activities, involved the harmonization and classification of the selected key performance indicators and their quantification to provide standard benchmarking values for examined ports. Fig 2 provides an overview of the process followed to produce the KPIs, the grading system used and final weighting to provide an overall value. Two different sets were produced focusing to differentiate purely passenger oriented ports from mainly freight ones.

\begin{tabular}{|c|c|c|c|c|}
\hline KPI Group & KPI Name & KPI No. & KPI Weight & Group Weight \\
\hline \multirow{7}{*}{ Traffic } & Annual number of ship calls & $\mathrm{KPI}_{11}$ & 0,20 & \multirow{7}{*}{0,24} \\
\hline & Average tonnage per ship & $\mathrm{KPI}_{12}$ & 0,15 & \\
\hline & Total annual throughput & $\mathrm{KPI}_{13}$ & 0,15 & \\
\hline & Annual number of TEUs & $\mathrm{KPI}_{14}$ & 0,20 & \\
\hline & Annual tonnage bulk commodities & $\mathrm{KPI}_{15}$ & 0,10 & \\
\hline & Annual tonnage non-bulk commodities & $\mathrm{KPI}_{16}$ & 0,10 & \\
\hline & Annual vehicle traffic (trucks) & $\mathrm{KPI}_{17}$ & 0,10 & \\
\hline \multirow{3}{*}{ Financial } & Business volume & $\mathrm{KPI}_{21}$ & 0,26 & \multirow{3}{*}{0,25} \\
\hline & Profitability & $\mathrm{KPI}_{22}$ & 0,49 & \\
\hline & Return on Capital Employed & $\mathrm{KPI}_{23}$ & 0,25 & \\
\hline \multirow{10}{*}{$\begin{array}{l}\text { Operational, } \\
\text { HR \& Other }\end{array}$} & EDI System & $\mathrm{KPI}_{31}$ & 0,08 & \multirow{10}{*}{0,19} \\
\hline & Single Window & $\mathrm{KPI}_{32}$ & 0,08 & \\
\hline & Port Community System & $\mathrm{KPI}_{33}$ & 0,08 & \\
\hline & Intermodal / Hinterland network & $\mathrm{KPI}_{34}$ & 0,15 & \\
\hline & Staff - Port Authority & $\mathrm{KPI}_{35}$ & 0,14 & \\
\hline & Staff - Port Community & $\mathrm{KPI}_{36}$ & 0,14 & \\
\hline & Tons per ship-hour in port & $\mathrm{KPI}_{37}$ & 0,10 & \\
\hline & Turn-around time (cargo) & $\mathrm{KPI}_{38}$ & 0,08 & \\
\hline & Waiting time after berthing for unloading & $\mathrm{KPI}_{39}$ & 0,07 & \\
\hline & Time waiting for cargo transfer between modes & $\mathrm{KPI}_{310}$ & 0,08 & \\
\hline Customs & Entry/ Exit Summary declaration (ENS/EXS) & $\mathrm{KPI}_{41}$ & 0,18 & 0,18 \\
\hline
\end{tabular}




\begin{tabular}{|llccc|}
\hline KPI Group & \multicolumn{1}{c}{ KPI Name } & KPI No. & KPI Weight & Group Weight \\
\hline Procedures & Electronic Customs Declaration (SAD) & KPI $_{42}$ & 0,18 \\
& $\begin{array}{l}\text { Economic Operation Registration \& } \\
\text { Identification (EORI) }\end{array}$ & KPI $_{43}$ & 0,14 \\
& Authorized Economic Operator (AEO) & KPI $_{44}$ & 0,24 \\
& Time required for goods clearance & KPI $_{45}$ & 0,26 \\
\hline Sustainability & Environmental Management System & KPI $_{51}$ & 0,45 \\
/ Security & Compliance with ISPS & KPI $_{52}$ & 0,55 & 0,14 \\
\hline
\end{tabular}

\section{Table 1 - Key Port Performance Indicators for Freight Activities}

As a result of the process, the resulting list of KPIs and weight for overall grading was obtained as provided in Table 1 and calculated for the 60 studied port. However, the lack of raw data, and the discrepancies in measurement methods and times among the ports forced to harmonize the values and/or complement data provided by the ports with desktop research. It was hence possible to capture the latest ranking status of the examined ports at a specific time (year 2012). Table 2 provides the aggregate KPI scoring for the freight ports studied.

At the end of the construction of the KPIs set it was recommended to develop a more robust benchmarking tool to obtain more reliable and exploitable findings either on a port's performance or when comparing different ports. 


\begin{tabular}{|c|c|c|c|c|c|c|c|c|c|c|c|c|c|c|c|c|c|c|c|c|c|c|c|c|c|c|c|c|c|c|c|}
\hline KPI Category & 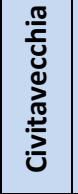 & 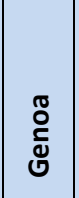 & 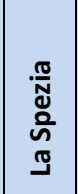 & $\begin{array}{l}\stackrel{0}{\grave{z}} \\
\stackrel{3}{3}\end{array}$ & 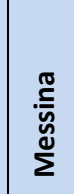 & $\frac{\frac{\tilde{y}}{\frac{0}{0}}}{\frac{\pi}{\tilde{\pi}}}$ & 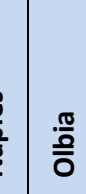 & 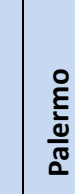 & & 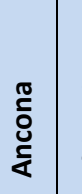 & 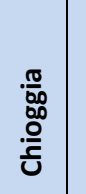 & 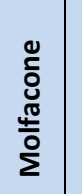 & 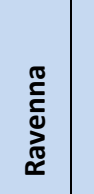 & 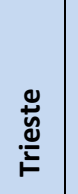 & 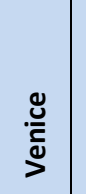 & 焉 & 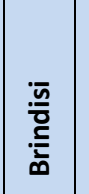 & $\begin{array}{l}\stackrel{0}{\frac{1}{5}} \\
\frac{\pi}{\circ} \\
\frac{\pi}{0} \\
\frac{0}{0}\end{array}$ & 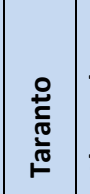 & 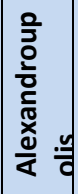 & 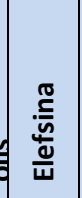 & 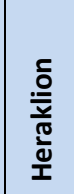 & 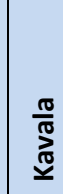 & 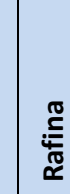 & & 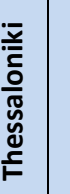 & 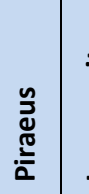 & 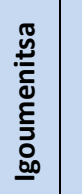 & $\frac{\check{\circ}}{\circ}$ & 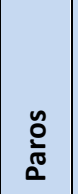 & 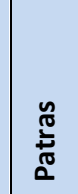 \\
\hline Traffic Indicators & 0,25 & 0,78 & 0,46 & 0,58 & 0,32 & 0,48 & 80,50 & 00,3 & & $\begin{array}{l}0,23 \\
0,\end{array}$ & $0,50: 0$ & $0,16 \mid c$ & $\begin{array}{lll}0,39 & 0 \\
\end{array}$ & 0,61 & $0,41 \mid \mathrm{c}$ & 0,21 & $0,21 \mid \mathrm{C}$ & 0,62 & 0,53 & 0,51 & 0,43 & 0,29 & 0,5 & 10,4 & &, $33 \mid 0$ & 0,550 & $0,74 \mid c$ & 0,22 & 0,54 & 0,37 \\
\hline Financial Indicators & 0,63 & 0,63 & 0,63 & 0,63 & 0,63 & 30,63 & $53 \mid 0,63$ & 30,6 & & 0,630 & \begin{tabular}{l|l}
0,63 & 0 \\
0
\end{tabular} & 0,63 C & \begin{tabular}{l|l}
0,63 & 0 \\
\end{tabular} & 0,63 & 0,63 c & 0,63 & 0,63 & 0,63 & \begin{tabular}{|l|c|c|}
0,63 & $c$
\end{tabular} & 0,00 & 0,39 & 0,63 & 0,0 & $0 \mid 0,1$ & $17 \mid 0$, &, $76 \mid 0$ & $0,34 \mathrm{c}$ & 0,50 & 0,19 & 0,63 & 0,46 \\
\hline Operational Indicators & 0,66 & 0,62 & 0,62 & 0,62 & 0,50 & 0,62 & $\left\{\begin{array}{l|l}2 & 0,48 \\
\end{array}\right.$ & 80,5 & & 0,610 & 0,560 & 0,62 c & \begin{tabular}{l|l}
0,62 & 0 \\
\end{tabular} & 0,63 & 0,63 & 0,55 & 0,55 & 0,40 & $0,57 \mid \mathrm{C}$ & 0,52 & 0,51 & 0,44 & 0,5 & 80,6 & &, 58 & \begin{tabular}{l|l}
0,66 & 0 \\
\end{tabular} & $0,51 \mid c$ & 0,55 & 0,40 & 0,37 \\
\hline Indicators for Customs Procedures & 0,78 & 0,78 & 0,68 & 0,68 & 0,68 & $80,6 \varepsilon$ & 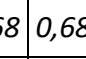 & 80,7 & & $\begin{array}{l}0,61 \\
0,\end{array}$ & \begin{tabular}{l|l}
0,61 & 0
\end{tabular} & $0,61 \mid 0$ & \begin{tabular}{l|l}
0,61 & 0
\end{tabular} & 0,68 & $0,61 \mid \mathrm{c}$ & 0,61 & $0,61 \mid \mathrm{c}$ & 0,61 & $0,61 \mid \mathrm{c}$ & 0,78 & 0,87 & 0,78 & 0,7 & 80,7 & $78 \mid 0$, &, $78 \mid 0$ & $\begin{array}{l}0,78 \\
0\end{array}$ & $0,88 \mid c$ & $0,78 \mathrm{C}$ & 0,78 & 0,67 \\
\hline Indicators for Sustainability/Security & 0,70 & 0,70 & 0,39 & 0,70 & 0,19 & 90,15 & 190,39 & 90,7 & & 0,70 o, & \begin{tabular}{l|l}
0,39 & 0
\end{tabular} & $0,39 \mid 0$ & \begin{tabular}{l|l}
0,39 & 0
\end{tabular} & 0,70 & $0,70 \mid c$ & 0,70 & $0,70 \mid c$ & 0,70 & $0,70 \mid c$ & 0,39 & 0,39 & 0,39 & 0,3 & 90,0 & $00 \mid 0$, &, $39 \mid 0$, & 0,700 & $0,70 \mid 0$ & $0,70 \mathrm{C}$ & 0,39 & 0,70 \\
\hline Total Performance Score & 3,01 & 3,51 & 2,76 & 3,20 & 2,31 & $2,6 c$ & 02,66 & 62,9 & & 2,772 & 2,682 & 2,402 & $2,63 \mid 3$ & $3,23:$ & $2,97: 2$ & 2,69 & $2,69: 2$ & 2,96 & $3,\left.04\right|^{2}-2 y$ & 2,20 & 2,59 & 2,53 & 2,2 & 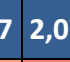 & $07 \mid 2$ & ,843, & 3,043 & \begin{tabular}{l|l}
3,34 & 2 \\
\end{tabular} & 2,442 & 2,74 & 2,57 \\
\hline$\%$ of Ideal Port & $60 \%$ & $70 \%$ & $55 \%$ & $64 \%$ & $46 \%$ & $52 \%$ & $\% 53 \%$ & $\% 60 \%$ & & $55 \% 5$ & $54 \% \mid 4$ & $48 \% 5$ & \begin{tabular}{|l|l}
$53 \%$ & 6 \\
\end{tabular} & $65 \%$ & $59 \%$ & $54 \%$ & $54 \%$ & $59 \%$ & $61 \%$ & $44 \%$ & $52 \%$ & $51 \%$ & $45 \%$ & 419 & & $\begin{array}{lll}7 \% & 6 \\
\end{array}$ & $51 \% 6$ & \begin{tabular}{l|l}
$57 \%$ & 4 \\
\end{tabular} & $49 \% 5$ & $55 \%$ & $51 \%$ \\
\hline KPI Category & 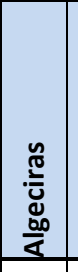 & 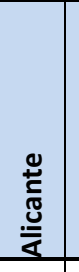 & $\begin{array}{l}\frac{\pi}{\bar{\Xi}} \\
\frac{\tilde{\varepsilon}}{\alpha} \\
\end{array}$ & 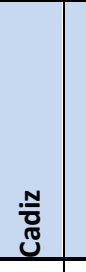 & 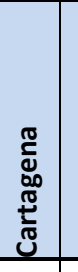 & 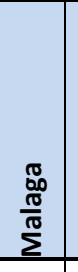 & 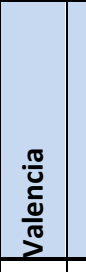 & 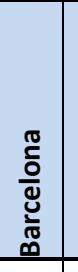 & 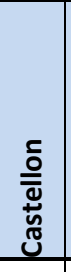 & \begin{tabular}{|l} 
\\
$\stackrel{\sqrt[N]{N}}{\underline{\underline{\underline{N}}}}$ \\
\end{tabular} & 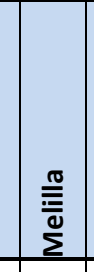 & 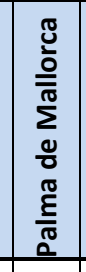 & 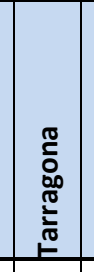 & $\stackrel{2}{\beth}$ & 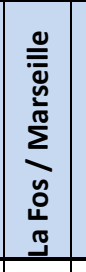 & ฆँ & 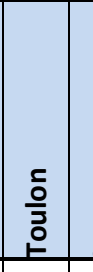 & 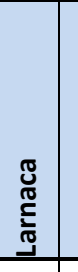 & 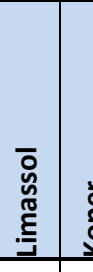 & 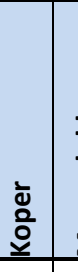 & 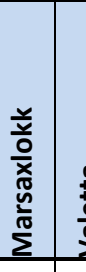 & 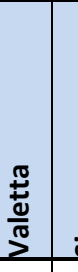 & 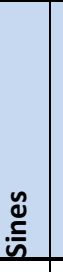 & 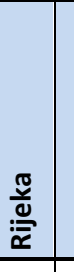 & 造 & 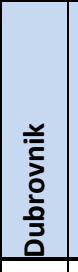 & 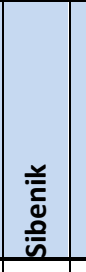 & 䓂 & \begin{tabular}{|l|} 
\\
$\frac{1}{5}$ \\
$\tilde{N}$ \\
\\
\end{tabular} & 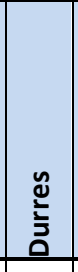 & $\frac{\check{0}}{\frac{0}{\nu}}$ \\
\hline Traffic Indicators & 0,99 & $0,07 \mathrm{C}$ & 0,100 & 0,080 & 0,43 & 0,13 & 0,830 & 0,88 & 0,27 & 0,19 & 90,15 & 0,35 & $50,47 \mathrm{C}$ & 0,31 & $0,81 \mathrm{C}$ & $0,13 \mathrm{C}$ & 0,160 & 0,090 & 0,190, & 0,400 & \begin{tabular}{l|l}
0,52 & 0, \\
\end{tabular} &, $18 \mid 0$ & , 58 & 0,160 & 0,13 & 0,21 & 0,27 & 0,210 & 0,10 & 0,08 & 0,20 \\
\hline Financial Indicators & 0,92 & 0,49 & 0,12 C & 0,570 & 0,89 & 0,43 & 0,930 & 0,98 & 0,29 & 0,37 & 70,26 & 0,37 & $70,89 \mathrm{C}$ & 0,47 & 0,79 & $0,63 \mathrm{C}$ & 0,500 & 0,620 & 0,700, & $0,85 \mid 0$, & $0,63 \mid$ & $\begin{array}{l}0,63 \\
\end{array}$ &, $83 \mid \mathrm{C}$ & 0,630 & 0,29 & 0,63 & 0,63 C & $0,63 \mid c$ & 0,63 & 0,83 & 0,63 \\
\hline Operational Indicators & 0,56 & 0,50 C & $0,46 \mid c$ & 0,450 & $0,57 \mathrm{C}$ & 0,44 & 0,640 & 0,61 & 0,51 & 0,39 & 90,29 & 0,54 & $40,71 \mathrm{C}$ & 0,62 & $0,60 \mid \mathrm{C}$ & 0,66 & 0,620 & 0,550 & 0,610, & \begin{tabular}{l|l}
0,72 & 0, \\
\end{tabular} & 0,59 , & 0,610 & $0,67 \mid c$ & 0,390 & 0,37 & 0,47 & 0,47 & $0,47 \mid c$ & $0,34 \mid c$ & 0,40 & 0,37 \\
\hline Indicators for Customs Procedures & 0,90 & $0,90 \mathrm{C}$ & 0,900 & 0,900 & 0,90 & 0,90 & 0,900 & 0,90 & 0,90 & 0,90 & 0,73 & 0,90 & 0,78 & 0,90 & 0,90 & 0,90 & 0,900 & 0,450 & 0,450 , & 0,780 & $0,57 \mid 0$, & 0,570 & 8,89 & $0,57 \mathrm{C}$ & 0,57 & 0,57 & $0,57 \mid$ & $0,57 \mid c$ & 0,57 & 0,78 & 0,57 \\
\hline Indicators for Sustainability/Security & 0,70 & $0,70 \mathrm{C}$ & $0,70 \mid c$ & 0,700 & 0,70 & 0,70 & 0,700 & 0,70 & 0,39 & 0,57 & 70,51 & 0,57 & 70,70 & 0,54 & 0,70 & $0,70 \mathrm{C}$ & $0,70 \mid 0$ & 0,700 & 0,700 , & $0,70 \mid$ & 0,390, & 0,390 &, 70 C & 0,70 C & 0,70 & 0,70 & 0,70 & $0,70 \mid c$ & $0,70 \mid \mathrm{c}$ & 0,70 & 0,70 \\
\hline Total Performance Score & 4,06 & $2,662^{2}-2$ & 2,282 & 2,703 & 3,492 & 2,60 & 4,004 & 4,07 & 2,36 & 2,43 & 31,94 & 2,74 & $4,5,52$ & 2,85 & $3,793^{3}$ & 3,022 & 2,882 & $2,41 \mid 2$ & 2,64 3, & \begin{tabular}{l|l}
3,46 & 2
\end{tabular} & 2,692 & $2,373^{3} \geq$ & 3,672 & 2,442 & 2,07 & 2,57 & 2,632 & $2,\left.57\right|^{2}-1-2$ & $2,332^{2}-x-2$ & 2,79 & 2,46 \\
\hline$\%$ of Ideal Port & $81 \%$ & $53 \% \mid$ & $46 \% 5$ & $54 \% 7$ & $70 \% 5$ & $52 \%$ & $80 \% 8$ & $81 \%$ & $47 \%$ & $49 \%$ & $639 \%$ & $55 \%$ & $671 \% 5$ & $57 \%$ & $76 \% \mid \epsilon$ & $60 \% 5$ & $58 \% 4$ & $48 \% 5$ & $53 \% 6 s$ & $9 \% 5$ & $54 \% \mid 4$ & $47 \% 7$ & $3 \%$ & $49 \%<$ & $41 \%$ & $51 \%$ & $53 \%$ & $51 \% / 4$ & $47 \% 5$ & $56 \%$ & $49 \%$ \\
\hline
\end{tabular}

Table 2 - Weighted Scores per KPI Category \& Total Performance Score for Freight Ports 


\subsection{Good Practices. Concept and identification}

Two separate questionnaires were distributed among the partners of the project and the 60 studied ports to identify the current good practices implemented to enhance the port operations and customs information processes. The lists produced provided an overview of what was considered to be well done.

In total, 301 good practices were identified. The results were processed, harmonized and classified following two distinct categorizations and, therefore, lists of good practices. The summarized lists were organized according to the process affected and the kind of solution used.

The first lists grouped the good practices according to "typology", being roughly based on the list of performance indicators for trade facilitation included in the WCO recommendations (World Customs Organization, 2014). Whereas the second list, was built around a list of 12 processes taking place during customs clearance produced by the partnership. As a result, Table 3 and Table 4 were produced.

\begin{tabular}{|c|c|}
\hline Best practices group & Practices identified \\
\hline $\begin{array}{l}\text { E-customs, } \\
\text { digitalization and } \\
\text { automation }\end{array}$ & $\begin{array}{l}\text { Computerized customs procedures; online clearance; reliable, fast and immediate } \\
\text { communication between administration bodies and importers; automatic clearance } \\
\text { and departure authorizations issuing (green risk goods); electronic customs } \\
\text { declaration (SAD); electronic accompanying export document (T2L); electronic } \\
\text { invoicing; check, ticketing and passenger control; generalized use of digital } \\
\text { certificates / signatures; no need of physical documents; software to facilitate the } \\
\text { generation of documentation like cargo manifests; automatic statistics record and } \\
\text { exploitation;... }\end{array}$ \\
\hline $\begin{array}{l}\text { Systematization, } \\
\text { homogenization, } \\
\text { simplification }\end{array}$ & $\begin{array}{l}\text { Coding systematization and simplification (i.e. aligning EORI with VAT, unique } \\
\text { vessel coding, TARIC adoption, etc.); centralized management of service requests } \\
\text { at port level; common encoding and language format for all declarations (XML); } \\
\text { joint (one-shop-stop) inspections by customs; border inspection health and } \\
\text { phytosanitary controls; simplified taxing levels; harmonized regulations;... }\end{array}$ \\
\hline $\begin{array}{l}\text { Information sharing } \\
\text { platforms. Port } \\
\text { communication \& } \\
\text { information exchange }\end{array}$ & $\begin{array}{l}\text { Single Window at port, national and regional level; wide use of Port Community } \\
\text { Systems by all stakeholders involved; custom inspection results sharing regarding } \\
\text { importers affected by all customs; use of EORI certification common to all EU } \\
\text { countries;... }\end{array}$ \\
\hline $\begin{array}{l}\text { Advance / Delayed } \\
\text { rulings }\end{array}$ & $\begin{array}{l}\text { Pre-arrival notifications; introduction of advanced rulings; possibility for advanced } \\
\text { clearance (applied to green risk goods and certified AEOs); post release audit }\end{array}$ \\
\hline $\begin{array}{l}\text { Risk management } \\
\text { and selective controls }\end{array}$ & $\begin{array}{l}\text { Automatic risk assessment; common risk criteria and assessment framework within } \\
\text { EU; emergency control centres and protocols of action; systematic check over } \\
\text { compliance with HSSE policies in terms of security, safety, pollution control and } \\
\text { health; risk assessment through ticketing (passengers); common framework for } \\
\text { AEO certification at EU; periodic checks on AEO certificated agents;... }\end{array}$ \\
\hline $\begin{array}{l}\text { Clearness, } \\
\text { transparency and } \\
\text { partnership with the } \\
\text { private sector }\end{array}$ & $\begin{array}{l}\text { Public access to complete list of customs procedures, fees and taxes; simplified } \\
\text { good levels and procedures; easy access to information regarding port services, } \\
\text { delays, procedures or other events through web services, SMS and/or social } \\
\text { networks; periodic meetings with actors involved; participation of all stakeholders } \\
\text { in decision making; periodic surveys to all stakeholders and publication of its } \\
\text { results; advisor boards; ... }\end{array}$ \\
\hline $\begin{array}{l}\text { Easy access to } \\
\text { multimodal } \\
\text { transportation }\end{array}$ & $\begin{array}{l}\text { Automated access systems; seamless connection to other modes of transportation; } \\
\text { Bonded warehousing; simplified connection and delayed procedures when working } \\
\text { with dry ports or logistic zones;... }\end{array}$ \\
\hline
\end{tabular}

\section{Table 3 - Best Practices according to typology ities}




\begin{tabular}{|c|c|}
\hline Procedure considered & Related good practices \\
\hline ENS declaration & $\begin{array}{l}\text { Online declarations lodging, use of XML standards and message structure defined } \\
\text { by DG TAXUD, digital signatures, measures to enforce compliance, automatic } \\
\text { risk analysis, make them universal by offering them for free... }\end{array}$ \\
\hline Arrival notification & $\begin{array}{l}\text { Use of SDTS declaration at the first port of entry as Arrival Notification, } \\
\text { submitted in advance, integration with Single Window / Port Community } \\
\text { Systems, share information between government authorities, use of one only } \\
\text { document for arrival notification, ENS, and good acceptance,... }\end{array}$ \\
\hline Risk identification & $\begin{array}{l}\text { All controls taken simultaneously when no high risk involved, common } \\
\text { framework at EU level, electronic certification of control automatically updated, } \\
\text { automatic risk sorting, algorithms to automatic identification of shipments with } \\
\text { higher risks, use of reliable operators with reduced controls, possibility of } \\
\text { advanced targeting to introduce clearance pre arrival, ... }\end{array}$ \\
\hline $\begin{array}{l}\text { Summary declaration } \\
\text { for temporary storage } \\
\text { (SDTS) }\end{array}$ & $\begin{array}{l}\text { Integration with Single Window at port / national level or Port Community } \\
\text { System, possibility of either multiple declarations (per consignee) or at vessel } \\
\text { level, use of proof of arrival message, consistency checks with other declarations } \\
\text { (ENS), control checks of cargo declared corresponds with cargo presented, } \\
\text { automatic countdowns and starting of goods departure (EXS), paperless } \\
\text { procedures, one declaration use for multiple purposes (ENS, arrival notification } \\
\text { and SDTS combined declaration),... }\end{array}$ \\
\hline $\begin{array}{l}\text { Electronic Customs } \\
\text { Declaration (SAD) }\end{array}$ & $\begin{array}{l}\text { Goods custom events history recording and traceability, simplified transit } \\
\text { procedures for containerized rail transport with dry ports, paperless procedures, } \\
\text { electronic official accompanying documents deemed valid, use of electronic } \\
\text { messages and digital signatures, ... }\end{array}$ \\
\hline $\begin{array}{l}\text { Export Accompanying } \\
\text { Document }\end{array}$ & $\begin{array}{l}\text { Electronic communication of goods arrival, automatic transmission of customs } \\
\text { events to port authority together with arrival notification, paperless control, } \\
\text { automatic and immediate exchange of all information by the different custom } \\
\text { offices affected,... }\end{array}$ \\
\hline $\begin{array}{l}\text { Exit Summary } \\
\text { Declaration (EXS) }\end{array}$ & $\begin{array}{l}\text { Online declarations lodging, use of XML standards and message structure defined } \\
\text { by DG TAXUD, digital signatures, measures to enforce compliance, automatic } \\
\text { risk analysis, make them universal by offering them for free, use of same massage } \\
\text { for transhipment whenever possible, use of the traceability system to inform Port } \\
\text { Authority of previous customs events, ... }\end{array}$ \\
\hline $\begin{array}{l}\text { Proof of Community } \\
\text { Status (T2L) }\end{array}$ & $\begin{array}{l}\text { Possibility to obtain it electronically, full paperless automatic procedure and } \\
\text { communication with the port of final destination, pdf (electronic) validity and no } \\
\text { need for hard copy documents, use of 2D/3D coding for security control, } \\
\text { integrated and common methodology at EU level,... }\end{array}$ \\
\hline $\begin{array}{l}\text { Customs Cargo } \\
\text { Manifest for Departure } \\
\text { (CCMD) }\end{array}$ & $\begin{array}{l}\text { Integrated in the Single Window, possibility to declare cargo either globally (for } \\
\text { whole vessel) or by consignee, automatic deadline settings for EXS or } \\
\text { transhipment documentations, automatic and paperless clearance and loading } \\
\text { controls for goods, ... }\end{array}$ \\
\hline $\begin{array}{l}\text { Economic Operator } \\
\text { Registration and } \\
\text { Identification (EORI) }\end{array}$ & $\begin{array}{l}\text { Use of the EORI figure, no coding duplicity at EU level, ease to calculation (use } \\
\text { of other coding as a base), use to speed up electronic processing and security } \\
\text { controls, ... }\end{array}$ \\
\hline $\begin{array}{l}\text { Authorised Economic } \\
\text { Operator (AEO) }\end{array}$ & $\begin{array}{l}\text { Use of the figure to reduce controls and optimization of resources elsewhere, } \\
\text { periodic updates to renovate the condition, common AEO listing at EU level, } \\
\text { equivalent standards to provide the certification, information exchange of } \\
\text { goodness of operators at EU level, benefits shared at all ports, ... }\end{array}$ \\
\hline $\begin{array}{l}\text { Goods release when } \\
\text { entering or leaving the } \\
\text { CTC }\end{array}$ & $\begin{array}{l}\text { Electronic submission of declarations, automatic risk analysis and filters, } \\
\text { reduction and automation of revenues, control to check quantities stored, cleared } \\
\text { and released, ... }\end{array}$ \\
\hline
\end{tabular}

Table 4 - Best practices according to the procedure affected

\section{METHODOLOGY. DEA ASSESSMENT}

\subsection{Good practices, compliance and grading. Approach taken}

Having identified both, available KPIs and two exhaustive lists of good practices, a nonparametric approach was proposed to link both concepts: problem a CRS, inbound oriented with slacks DEA, despite VRS was also tested as a possible method of resolution. The approach 
has two main strengths: the detection of the peers (or the dominating ports) that can serve as reference for performance improvement to other ports and, moreover, the various decompositions of the overall efficiency can point towards more specific means to improve efficiency, i.e. what practices are likely to provide a higher performance boost if fulfilled.

DEA is useful when the production process presents a structure of multiple inputs and outputs. Unlike traditional regression analysis (what was pretended to be used at the beginning), DEA does not assume a starting production function between inputs and outputs, but constructs a piecewise linear line which represents the production frontier. The frontier links the best performers regarding input inverted and output obtained. This "best performers" are known as peers of the DEA analysis.

Regarding the specifities of the DEA approach, the previous works in port benchmarking and the specificities of the problem, a CRS, inbound oriented with slacks DEA was used as a base for the assessment of the good practices, despite VRS was also tested as a possible method of resolution. The difference between the CRS and VRS models is on the allowed variables for calculus, so while the former provides information purely on technical and scale efficiency, the latter identifies technical efficiency alone.

Despite that, it is known that DEA has some drawbacks of its own. Moreover, in this case, some extra drawbacks were identified: the inputs finally used were far from representative considering the outputs and both (inputs and outputs) were more qualitative than quantitative values since relative (and weighted) values were finally taken. However, the main alternative in terms of benchmarking, SFA, obliged to define a particular frontier shape and to make assumptions on the distributions of the errors which was not possible.

The results obtained then, cannot be taken straightforward and must be interpreted accordingly. The knowledge of the drawbacks plus the use of slacks helps interpreting the results and assist in the goal of this paper which is not to assess the ports but the good practices, a goal partially achieved.

\subsection{Particularities of the methodology}

One of the drawbacks of DEAs with Farrell approach is that the ports can have an efficiency score of 1 and still be inefficient, since some inputs could be reduced or some outputs could be expanded without affecting the need for other inputs or outputs. The phenomenon is quite common on practical DEA applications, and the distances we can reduce the inputs (or increase the outputs) without leaving the frontier isoquant are known as slacks (Bogetoft and Otto, 2010).

To account for the slacks it is possible to include to the formulation some penalization to the Farrell efficiency of ports that have them. Therefore, the efficiency values in section 5 of this deliverable are given without slacks and also after penalizing in 5\% the existence of slacks both in the inputs are output sides of the port.

Additionally, the relationship between the ports results and the ports that are being considered 
as "efficient" or belonging to the production frontier can be parametrized. Therefore, from checking their values it is possible to identify what ports are really a reference to most of the other studied ports, and from there, considering the current practices in the most referenced ports assess what are the most efficient practices.

\subsection{Inputs and outputs definition}

The specification of variables to be used is critical. Specifying erroneous or unfit inputs or outputs may cause biased results and conclusions. This is especially true in this case, where the lack of quality data in some cases might have an adverse effect on the efficiency estimates. Besides, increasing the number of dimensions (variables) used, reduces the discriminatory power of the technique, resulting in increasing measured efficiency and consequently increasing the number of ports being identified as fully efficient. In such scenario, the chance that a port is deemed different from its peers increases and as a result the port may be deemed efficient only due to the lack of comparator observations.

There is a rule of thumb establishing that the maximum relationship between the number of observations (in this case ports) and the number of inputs and outputs. According to Bogetoft and Otto (2010) the number of observations, K, should comply $K>3(m+n)$ and $K>m \cdot n$, to have meaningful results, with $m$ and $n$ the number of inputs and outputs. As a result, the ideal selection includes the smallest number of output and input measures that adequately capture all essential aspects of a port's operations and this would be in conjunction with the number of ports used in the model. Hence, a key issue for researchers is at what level of aggregation inputs/outputs should be established.

Besides that, inputs and outputs are usually taken as comparable inverted units per production units obtained in return. In the case of ports, the existing literature uses, among others, labour, equipment (units or costs), handling operations, berth length as inputs and throughput traffic, TEU berth hours or revenue as outputs of the model (see Tovar \& Rodríguez-Déniz, (2015) or Panayides, Maxoulis et al. (2009) for complete listings on the variables used thus far in ports DEA literature).

In this case, ideally, outputs and inputs were to be selected from the KPIs, and the efficiency values obtained to be correlated to the completeness of each set of good practices. However, since the data was not sufficiently complete, the level of implementation of the identified good practices was finally taken as inputs.

From Table 3 and Table 4 a total of 43 and 84 aspects were considered individually regarding the 8 and 12 groups of good practices. In total, 117 good practices -some of them shared in both listings and/or two different chapters from the same list- were ranked individually for each of the 60 ports analyzed, the marks given going from 0 to 1 , being 1 a $100 \%$ implementation mark and 0 an inexistent practice in the port in consideration. The values where obtained directly from the data collected during the study. But in some cases the good practices could not be directly rated with the information provided. In such cases the values could usually be assumed implicitly from the reports and questionnaires and some desk work. 
Since, the 43 and 83 are far too much practices, the gradings were grouped together for each group of practices, reaching 8 and 12 values in total. This should allow a straightforward interpretation on the role of each set to the global efficiency for each of the peers.

Regarding the outputs, 27 are too many KPIs to be used as outputs, too many variables for a too short array of ports (40 with a sufficient amount of data). Therefore, it is necessary to either reduce the current list of KPIs to a manageable number or use aggregate KPI values similarly of what has been done with the definition of the inputs. As a result there were two meaningful approaches available:

- Taking the normalized and weighted scorings for each set of KPIs (traffic, financial, operational and HR, customs, and sustainability/security sets) as shown in Table 1 to a final total of 5 outputs. Alternatively, the final weighted value could be used to produce only one final output.

- To consider the KPIs related to port performance that are available for a majority of the ports studied, that is, to consider the 7 traffic indicators. In this scenario the values used could be in their absolute form (before normalizing).

\subsection{Considered ports}

Data was obtained for 60 ports, given the limited completeness in some cases, two thresholds was used. Only ports with data fulfilling both conditions were taken into account (40 in total):

- Ports with real data in at least 4 of the 7 traffic indicators (KPIs) (43 ports in total).

- Ports with over $50 \%$ of the good practices (58 over 117) described either implicitly or explicitly and by means of any of the sources described previously, i.e. questionnaires and reports (55 ports considered).

\section{RESULTS}

In total, sixteen combinations of ports, inputs and outputs were studied, considering a varying number and type of inputs, outputs and list of ports. Only the results for the final selection of ports (40) and an aggregated and weighted final KPI value (output) and aggregated good practices (inputs) are being discussed here.

\subsection{DEA results}

The more distinct values are obtained when using the absolute -raw- values used to calculate the traffic KIPs either, when considering the good practices grouped by typology or customs procedure. However, this combination resulted in more difficult results to interpret, regarding the relative importance of the different sets of good practices considered (which in fact is the goal of the research), in such case the easier results to use are the ones with normalized input and outputs.

\subsection{Port efficiency}

The efficiency values are calculated following the Farrell's efficiency approach considering slack of 5\% (distance allowed to reduce the inputs or increase the outputs) without leaving the frontier isoquant). 
Efficiency values close to $100 \%$ indicate that a port is taking the maximum advantage of its current inputs (good practices) comparatively with the rest of ports studied. In fact, a 100 value indicates that a proportional reduction in all inputs considered would mean a reduction on the outputs. That is, the port is taking the maximum advantage of at least one input.

After calculating the Farrell efficiency on the selection of 40 ports with the slack, Table 5 is obtained showing the efficiency of each port, considering the level of implementation of the good practices (using both classifications proposed) with and without slacks.

\begin{tabular}{|c|c|c|c|c|c|c|}
\hline & \multicolumn{3}{|c|}{ EFFICIENCY (TYPE) } & \multicolumn{2}{|c|}{ EFFICIENCY (PROC) } & \multirow[b]{2}{*}{ Slack? } \\
\hline & Original & Modified & Slack? & Original & Modified & \\
\hline Civitavecchia & $73 \%$ & $65 \%$ & TRUE & $52 \%$ & $36 \%$ & TRUE \\
\hline Genoa & $100 \%$ & $100 \%$ & FALSE & $95 \%$ & $74 \%$ & FALSE \\
\hline Livorno & $100 \%$ & $100 \%$ & FALSE & $75 \%$ & $58 \%$ & FALSE \\
\hline Messina & $85 \%$ & $78 \%$ & TRUE & $54 \%$ & $40 \%$ & TRUE \\
\hline Palermo & $82 \%$ & $75 \%$ & TRUE & $53 \%$ & $39 \%$ & TRUE \\
\hline X.Ancona & $80 \%$ & $70 \%$ & TRUE & $52 \%$ & $39 \%$ & TRUE \\
\hline Molfacone & $83 \%$ & $70 \%$ & TRUE & $52 \%$ & $38 \%$ & TRUE \\
\hline Ravenna & $94 \%$ & $79 \%$ & TRUE & $62 \%$ & $50 \%$ & TRUE \\
\hline Trieste & $100 \%$ & $100 \%$ & FALSE & $77 \%$ & $60 \%$ & FALSE \\
\hline Venice & $89 \%$ & $82 \%$ & TRUE & $63 \%$ & $52 \%$ & TRUE \\
\hline Bari & $67 \%$ & $55 \%$ & TRUE & $52 \%$ & $38 \%$ & TRUE \\
\hline Brindisi & $71 \%$ & $59 \%$ & TRUE & $52 \%$ & $39 \%$ & TRUE \\
\hline Taranto & $90 \%$ & $86 \%$ & TRUE & $86 \%$ & $64 \%$ & TRUE \\
\hline Heraklio & $100 \%$ & $100 \%$ & FALSE & $100 \%$ & $96 \%$ & FALSE \\
\hline Thessaloniki & $100 \%$ & $96 \%$ & TRUE & $100 \%$ & $96 \%$ & TRUE \\
\hline Piraeus & $99 \%$ & $91 \%$ & TRUE & $100 \%$ & $100 \%$ & TRUE \\
\hline Volos & $100 \%$ & $95 \%$ & TRUE & $100 \%$ & $96 \%$ & TRUE \\
\hline Patra & $100 \%$ & $100 \%$ & FALSE & $100 \%$ & $100 \%$ & FALSE \\
\hline Algeciras & $100 \%$ & $100 \%$ & FALSE & $100 \%$ & $100 \%$ & FALSE \\
\hline Alicante & $75 \%$ & $58 \%$ & TRUE & $54 \%$ & $39 \%$ & TRUE \\
\hline Almeria & $75 \%$ & $60 \%$ & TRUE & $54 \%$ & $39 \%$ & TRUE \\
\hline Cadiz & $75 \%$ & $60 \%$ & TRUE & $54 \%$ & $39 \%$ & TRUE \\
\hline Cartagena & $82 \%$ & $75 \%$ & TRUE & $64 \%$ & $52 \%$ & TRUE \\
\hline Malaga & $75 \%$ & $59 \%$ & TRUE & $54 \%$ & $39 \%$ & TRUE \\
\hline Valencia & $84 \%$ & $79 \%$ & TRUE & $90 \%$ & $86 \%$ & TRUE \\
\hline Barcelona & $87 \%$ & $80 \%$ & TRUE & $93 \%$ & $90 \%$ & TRUE \\
\hline Castellon & $78 \%$ & $67 \%$ & TRUE & $55 \%$ & $39 \%$ & TRUE \\
\hline Eivissa & $88 \%$ & $77 \%$ & TRUE & $54 \%$ & $39 \%$ & TRUE \\
\hline Melilla & $100 \%$ & $88 \%$ & TRUE & $54 \%$ & $40 \%$ & TRUE \\
\hline Palma_Mallorca & $84 \%$ & $77 \%$ & TRUE & $59 \%$ & $45 \%$ & TRUE \\
\hline Tarragona & $72 \%$ & $65 \%$ & TRUE & $66 \%$ & $55 \%$ & TRUE \\
\hline Marseille & $100 \%$ & $100 \%$ & FALSE & $100 \%$ & $100 \%$ & FALSE \\
\hline Larnaca & $100 \%$ & $83 \%$ & TRUE & $73 \%$ & $44 \%$ & TRUE \\
\hline Limassol & $100 \%$ & $85 \%$ & TRUE & $69 \%$ & $43 \%$ & TRUE \\
\hline Koper & $100 \%$ & $100 \%$ & FALSE & $100 \%$ & $100 \%$ & FALSE \\
\hline X.Rijeka & $100 \%$ & $100 \%$ & FALSE & $100 \%$ & $100 \%$ & FALSE \\
\hline Ploce & $100 \%$ & $100 \%$ & TRUE & $100 \%$ & $97 \%$ & TRUE \\
\hline Zadar & $100 \%$ & $99 \%$ & TRUE & $100 \%$ & $99 \%$ & TRUE \\
\hline Durres & $100 \%$ & $100 \%$ & FALSE & $100 \%$ & $100 \%$ & FALSE \\
\hline Vlore & $100 \%$ & $100 \%$ & FALSE & $100 \%$ & $100 \%$ & FALSE \\
\hline
\end{tabular}

Table 5 - Farrell efficiency for the selection of 40 ports with and without slacks

Thus far, the results show how well ports are performing considering the amount of good practises being implemented. That is, a smaller efficiency value does not mean a port is 
performing worse than another, it can just mean that it has more efficiency measures implemented than another. In fact, and at this level of the assessment it would be highly profitable to produce the assessment with panel data, considering the rates of several years.

The higher performers in absolute values, reach smaller efficiency values because of having a larger number of good practices implemented (high input values). This means that there is not a linear relationship between the level of completeness of each set of good practices and the performance (traffic indicators) and that it is likely that for this specific set of data the scalability is negative.

Regarding procedures versus typology sets of good practices, the former provide more distinct values than the latter, which means that the differentiation among ports is easier in that case. Therefore, there is more correspondence between the sets of good practices and the port performance when they are organized by sets according to customs and clearance procedures, meaning that this approach is preferable, at least for this assessment.

\subsection{Peers considered}

At the final stage of the analysis of ports performance, it is the moment to identify which peers are the most referenced, and therefore are the "real" benchmarks in terms of performance when considering the good practices implemented. This is achieved by checking the values given to the lambdas from the DEA assessment, as provided in Table 6 and Table 7

The results show how some peers are used by a small amount of ports (or even one), meaning that those ports are not representative, since they cannot be compared to any port but themselves. Additionally some other peers are used exclusively by several ports, meaning that those ports could interchange positions with the peer being considered, or that both perform equally.

It is also observed that there is a similar behaviour country-wise either in efficiency and/or the peers considered. This reflects how the data was gathered and that some values, especially in terms of good practices were constant among the ports of the same country. In fact, a further analysis of the results show a clear clustering country-wise, that is, when taking together only the ports of one specific country, the relative values regarding efficiency do not vary and slightly change. 


\section{Table 6 - Peer ports, and lambda values for good practices aggregated by procedures}

Peer

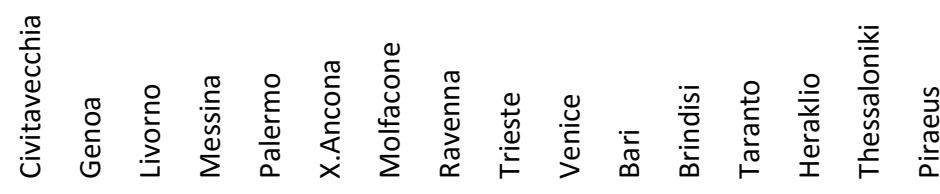

L Piraeus $\begin{array}{llllllllllllll}0 & 0,1 & 0,1 & 0,3 & 0,3 & 0 & 0 & 0,1 & 0,1 & 0,1 & 0 & 0\end{array}$

L_Patra $\begin{array}{llllllllllllll}0,3 & 0 & 0 & 0 & 0 & 0,3 & 0,3 & 0 & 0 & 0 & 0,3 & 0,3 & 0\end{array}$

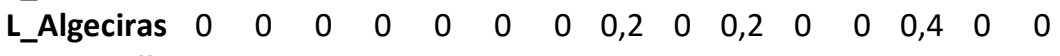

Marseill

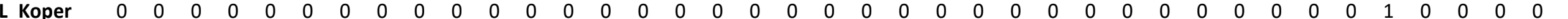

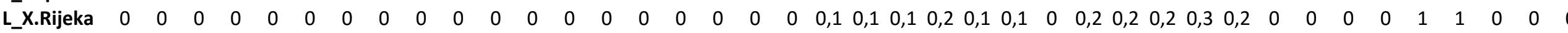

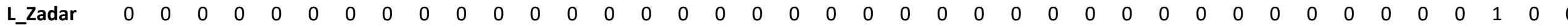

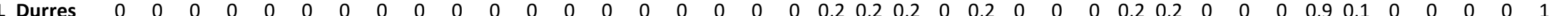

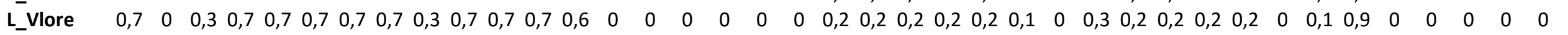

Table 7 - Peer ports, and lambda values for good practices aggregated by type of practice

Peer<smiles>O=C(O)OC(=O)O</smiles><smiles>CC(C)C(=O)O</smiles>

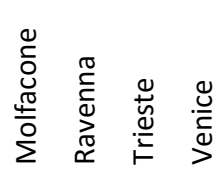

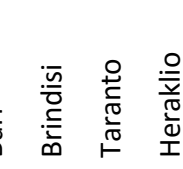

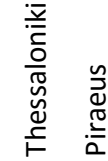

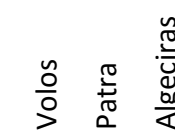

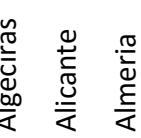

L Livorno

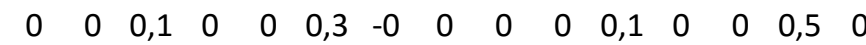

\section{$\begin{array}{lll}0 & 0 & 0\end{array}$} 00

L_Trieste

L_Heraklio $\begin{array}{lllcccc}0 & 0 & 0 & 0 & 0 & 0\end{array}$

L_Patra

$\begin{array}{ccccccccccccc}0 & 0 & 0 & 0 & 0 & 0 & 0 & 0 & 1 & 0,5 & 0 & 0 & 0,2 \\ 0 & 0 & 0 & 0 & 0 & 0 & 0 & 0 & 0 & 0 & 0 & 0 & 0 \\ 0 & 0 & 0 & 0 & 0,5 & 0 & 0 & 0,1 & 0 & 0 & 0 & 0 & 0\end{array}$

L_Algeciras

L_Marseille $\begin{array}{lllllll}0 & 0 & 0 & 0 & 0,5 & 0\end{array}$

$\begin{array}{ll}0 \\ 2 & 0\end{array}$

$\begin{array}{ll}0 & 0 \\ 0 & 0\end{array}$

L_Koper $\begin{array}{lllllllcl}0 & 0 & 0 & 0 & 0 & 0 & 0 & 0 & 0\end{array}$

L_X.Rijeka 00 $\begin{array}{lll}0 & 0 & 0\end{array}$

L_Durres $\begin{array}{cccccc}0 & 0 & 0 & 0 & 0 & 0 \\ 0 & 0 & 0 & 0,6 & 0,5 & 0,8\end{array}$ $\begin{array}{llllllll}0 & 0 & 0 & 0 & 0 & 0 & 0,1 & 0\end{array}$ $\begin{array}{lll}0 & 0,5\end{array}$

$\begin{array}{ll}0 & 0 \\ 0 & 0\end{array}$ $\begin{array}{lllll}0 & 0 & 0 & 0 & 0\end{array}$ 0 $\begin{array}{lll}0 & 0 & 0 \\ 0 & 0 & 0\end{array}$ 0
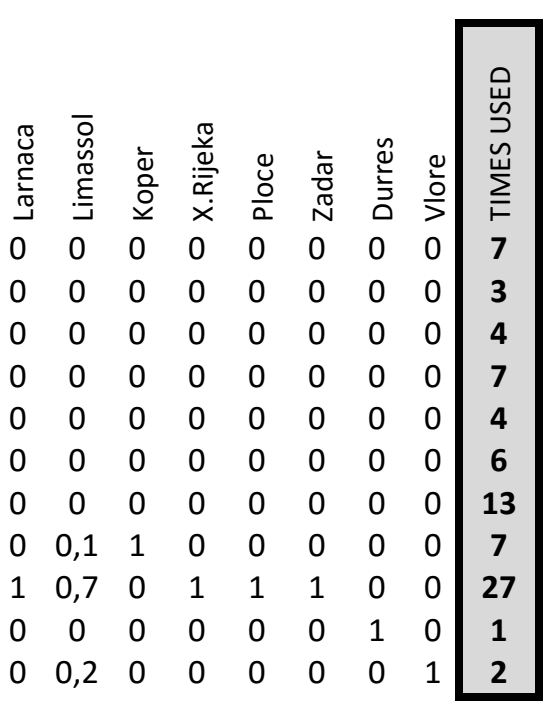


\section{DISCUSSION AND CONCLUSIONS}

\subsection{Overview}

The goal of this paper was to identify the best practices currently in operation in the Mediterranean and that might compose the concept of "ideal port".

First, a common framework of KPIs and Best Practices available was produced, assessing individually and practice by practice what is the level of compliance with the good practices identified for each of the 60 ports studied and considering 113 good practices in total.

In a second stage, an input oriented DEA with constant returns to scale and using the lambda values as assessment was used. The original list of 60 ports was shortlisted to 40 to include those with the most complete data set.

In total 16 models were developed from considering different combinations of variables and the results of 2 of them were discussed in more detail, using the normalized weighted values for the traffic indicators as output (only one variable) and the normalized good practices indicators (by set) as input variables. The two models discussed, were differentiated on the way the inputs were organized: good practices organized regarding procedures or regarding typology of the practice. The best results were obtained when the good practices were organized by port procedure.

\subsection{Conclusions and recommendations}

In terms of data, the KPIs and good practice listings are a good starting port for port evaluation and assessment. However, the existing inconsistencies on the data used, mainly due to incomplete data series and the lack of data evolution on time could be addressed with sustained updates on the database to build a comprehensive data panel analysis.

Regarding the assessment produced, a similar behaviour has been observed country-wise either in efficiency and/or the peers considered. This reflects certain -otherwise understandable- homogeneity on the measures implemented in each country, making advisable to produce recommendations at a country level as well as European-Mediterranean level. In that sense, it is recommended to directly use the KPIs as indicators when comparing ports at the Mediterranean level, and the efficiency obtained after the DEA analysis when comparing them at a national level.

Considering the assessment provide in this deliverable, the assessment would highly benefit from a consistent database with panel data registered over time more than a vast crosssectional data for a certain time period. This could be achieved from maintaining a port observatory operational for several years.

The ideal assessment of the good practices would have come from using quantifiable outputs 
related to the customs and clearance operations (e.g. average time for clearance, number of processes involved, average number of inspections) at a terminal level and investment effort (e.g. staff, equipment investment, etc) at an input level. At a second stage the effectiveness of the good practices would have been obtained after correlating the efficiency of this new DEA assessment and the level of completeness of the good practices implemented or, otherwise, the level of completeness could be introduced as another input as well.

To summarize, a database with historical registers keeping track of the inputs directly related to customs and clearance performance following common guidelines at an European level is necessary to identify the exact role of each good practice. The use of a Port Observatory would help in this course of action and better results are expected after using its database after some years of operation.

\section{REFERENCES}

BICHOU, K., GRAY, R. (2004). A Logistics and Supply Chain Management Approach to Port Performance Measurement. Maritime Policy and Management, 31(1), pp. 47-67.

BOGETOFT, P., OTTO, L. (2010). Benchmarking with DEA, SFA, and R, 1st ed. Springer, doi:0.1007/978-1-4419-7961-2

COOPER, W.W., SEIFORD, L.M., TONE, K. (2007). Data envelopment analysis: A comprehensive text with models, applications, references and DEA-solver software: Second edition, Springer Science+Business Media, LLC, doi:10.1007/978-0-387-45283-8

CULLINANE, K., Wang, T.-F., 2006. The efficiency of European container ports: A crosssectional data envelopment analysis. Int. J. Logist. 9, 19-31. doi: $10.1080 / 13675560500322417$

MARLOW, P.B., PAIXAO, A.C. (2003). Measuring lean ports performance. International Journal of Transport Management, 1, pp. 189-202.

OWINO, E.L., WANG, T.-F., PASUKEVICIUTE, I. (2006). Performance measurement in European container terminal industry: an empirical investigation, in: IAME 2006 Proceedings, 12-14 July, Melbourne, Australia.

PANAYIDES, P.M., MAXOULIS, C.N., WANG, T., NG, K.Y.A. (2009). A Critical Analysis of DEA Applications to Seaport Economic Efficiency Measurement. Transport Reviews, 29(2), pp. 183-206.

TALLEY, W.K. (1994). Performance indicators and port performance evaluation. Logistics and Transportation Review, 30(4), pp. 339-352.

TONGZON, J.L. (1995). Determinants of port performance and efficiency. Transportation Research. Part A: Policy and Practice. 29(3), pp. 245-252.

TOVAR, B., RODRÍGUEZ-DÉNIZ, H. (2015). Classifying Ports for Efficiency 
Benchmarking: A Review and a Frontier-based Clustering Approach. Transportation Reviews, 35(3), pp. 378-400.

UNCTAD (1976). Port performance indicators (TD/B/131/Supp.1/Rev.1). New York.

WORLD CUSTOMS ORGANIZATION (2014). Performance Indicators for the Agreement

on Trade Facilitation. (http://www.wcoomd.org/en/topics/wco-implementing-the-wtoatf/ /media/B35F6564E51D46B0AEF623C60BC47F50.ashx). Accessed on July 2015. 